\title{
Efficient Power Harvesting from the Mobile Phone Audio Jack for mHealth Peripherals
}

\author{
Chengyang Yao, Alexander Sun, and Drew A. Hall \\ Department of Electrical and Computer Engineering \\ University of California, San Diego \\ La Jolla, CA, USA
}

\begin{abstract}
This paper describes the design of a circuit to efficiently harvest energy from the standard audio jack of a mobile phone to power mHealth peripherals, lowering cost, reducing size, and improving practicality of portable medical devices. Not only does this design obviate the need to charge or change a battery, the presented circuit is also universally compatible with all phones. The audio output channels of several popular phones were characterized in order to determine the range of design parameters. Using these data, different power harvesting topologies are presented, simulated, and compared. A PCB implementation was used to measure and confirm the performance. Compared with prior art, which have achieved $21 \%$ and $47 \%$ efficiency, this design is able to achieve greater than $77 \%$ efficiency by using a tunable impedance matching network.
\end{abstract}

Keywords—smartphone; power harvesting; phone peripherals; impedance matching

\section{INTRODUCTION}

The 21 st century has witnessed a rapid growth of mobile phone usage. More than one-quarter of the global population currently uses a smartphone [1]. Almost unthinkably, more people today have access to a cell phone than to a clean toilet [2]. This wide spread access to mobile computing has inspired a surge of peripheral devices that add functionality to the mobile phone. With the processing power available on these now ubiquitous devices, mobile phone-based platforms present a tremendous opportunity to enable new applications in fields like mobile Health (mHealth) and Body Area Networks (BAN), providing an effective way to democratize healthcare worldwide. Many medically relevant sensors such as electrocardiograms (ECG), pulse oximeters, and molecular sensors can be integrated with a mobile phone to transform it into a specialized and portable medical device. By leveraging the widespread availability and high computational power of mobile technology, phone-based mHealth tools can be created to be more cost-effective and practical than their stand-alone counterparts. Such devices can bring much needed resources usually only available in well-equipped hospitals or centralized laboratories to individuals in remote or developing areas.

While the most obvious interface between a phone and a peripheral device would be the phone's own proprietary port, connection standards vary between different makes and models, making a device which is designed specifically for one port incompatible with others. For example, devices like the iBGStar blood glucose meter, which connects through Apple's 30-pin connector, cannot interface with Android, Windows, Blackberry, or feature phones. The only truly common interface

This work was partially supported by the Qualcomm Institute summer undergraduate research scholars program and the National Institutes of Health, Grant UL1TR000100. The content is solely the responsibility of the authors and does not necessarily represent the official views of the NIH.

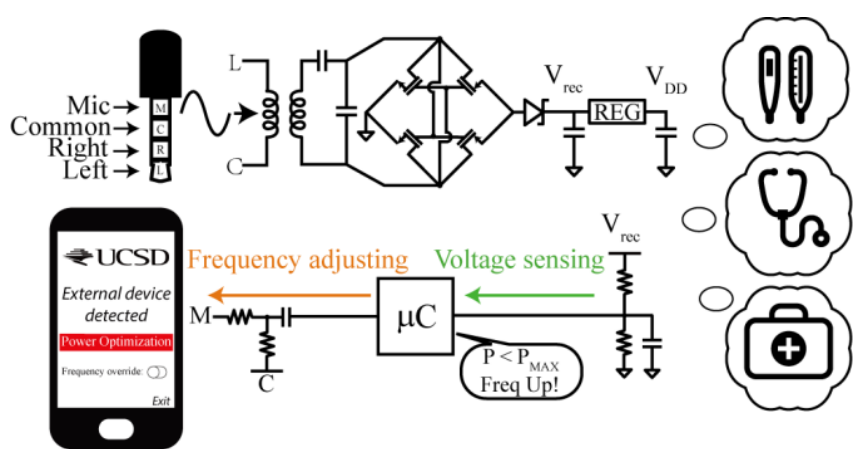

Fig. 1. Overview of system. The power harvester transforms, rectifies, and regulates a tone from the phone, whereas the microcontroller senses and optimizes the efficiency by adjusting he tone frequency generated by the mobile phone.

among all mobile phones is the headset audio port. Several smartphone accessories such as the Square and PayPal Credit Card reader have already utilized this ubiquitous access port for communication, but they still require batteries to supply power. In general, the inclusion of a battery not only increases the size and weight of the external peripheral but also limits the usage and convenience of the product, since over time batteries need to be recharged or replaced. Previous works such as the "Hijack" project [3], work of Laksanasopin et al [4] and "NXP Smartphone Quick-Jack solution" [5] have explored the feasibility of utilizing this audio interface both to power and communicate with external peripherals. However, the variation between the audio drivers in different phones poses a challenge for such systems to be generalizable [6]. Current topologies do not have sufficient efficiency to support more complex and power consuming applications and cannot adapt to the audio port variations of different phones. This paper will take a closer look at the audio interface and propose a solution to efficiently harvest power that is generalizable for all major smartphones and feature phones.

To this end, we have characterized the available power from the audio port of several popular smartphones, designed the circuit to harvest power augmented with an impedance matching network, and evaluated its efficiency. As illustrated in Fig. 1, there are four terminals in a standard $3.5 \mathrm{~mm}$ audio connector: the left and right output channels, common, and microphone input. Our power harvester uses the left channel to extract energy, while the right channel and the microphone are reserved for communication. Since each port is AC coupled with the exception of the microphone bias, which unfortunately can only provide very limited power [6], the phone is unable to directly 
supply a steady DC voltage. Instead, a tone is generated by the phone and converted to a usable DC voltage by external circuitry. The presented design also includes an impedance matching network and a software-hardware hybrid feedback loop to adjust the frequency to achieve optimal efficiency across a wide variety of phones with varying output impedances.

\section{PHONE CHARACTERIZATION}

Before any design can be undertaken, it is first necessary to determine the key specifications of each phone, namely: the maximum available power $\left(P_{\mathrm{AV}}\right)$ when the load impedance is matched with the phone, the output impedance $\left(R_{\mathrm{S}}\right)$, and the open-circuit output voltage $\left(V_{\mathrm{S}}\right)$. These characteristics are measured by applying a resistive load between the left channel and common terminal. By first sweeping the frequency, and then the load resistance, an effective circuit model for the phones can be determined. When the load is constant, changing the frequency from $20 \mathrm{~Hz}$ to $20 \mathrm{kHz}$, which is the typical audio range, results in less than $2.5 \%$ variation in the output voltage for all the phones tested. From this observation, it can be concluded that the output impedance is independent of the frequency and can be modeled as a constant internal resistance. Fig. 2 shows the available power curves generated by sweeping the load resistance and Table I summarizes the characteristics for each phone tested. In this paper, efficiency is defined as the ratio of power delivered to the load after rectification over the available power characterized by these data.

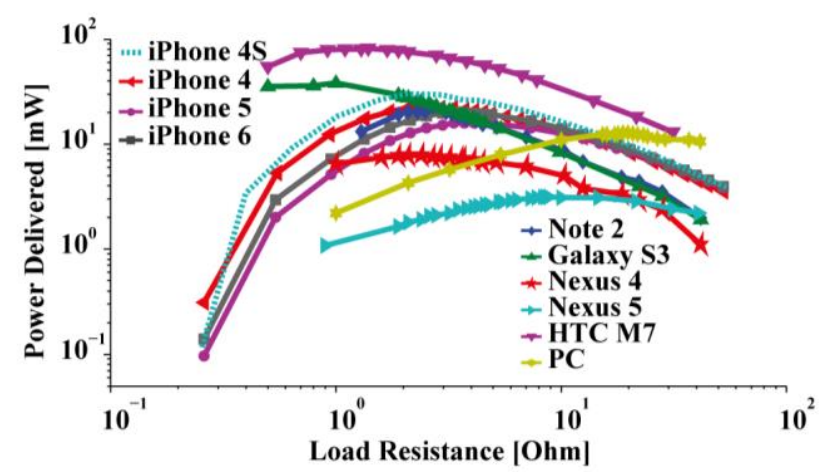

Fig. 2. Measured available power versus load curves.

TABLE I. SUMMARY FOR DIFFERENT PHONES

\begin{tabular}{|c|c|c|c|}
\hline Phone model & $\boldsymbol{V}_{\mathbf{S , R M S}}(\mathbf{m V})$ & $\boldsymbol{R}_{\mathbf{S}}(\boldsymbol{\Omega})$ & $\boldsymbol{P}_{\mathbf{A V}}(\mathbf{m W} \mathbf{)}$ \\
\hline iPhone 4 & 449 & 2.12 & 22.21 \\
\hline iPhone 4S & 471 & 3 & 23.69 \\
\hline iPhone 5S & 478 & 2.95 & 20.38 \\
\hline iPhone 6 & 479 & 3.72 & 19.89 \\
\hline Note 2 & 671 & 3.73 & 19.98 \\
\hline Galaxy S3 & 392 & 1 & 38.3 \\
\hline Nexus 4 & 256 & 2.1 & 7.77 \\
\hline Nexus 5 & 322 & 8.3 & 3.13 \\
\hline HTC M7 & 672 & 1.4 & 80.64 \\
\hline PC & 1,008 & 19.9 & 12.8 \\
\hline
\end{tabular}

A few interesting observations were made while testing this batch of devices. Firstly, as the battery level decreases from $100 \%$ to $1 \%$, the change in $V_{\mathrm{S}}$ is negligible across all phones. Secondly, for most phones, $R_{\mathrm{S}}$ is between $2 \Omega$ and $4 \Omega$, but $V_{\mathrm{S}}$ varies greatly from phone to phone, resulting in a wide range of available power. As a point of reference, power requirements for different medical devices is summarized in Table II. While some phones have more than enough power for all these sensors, unsatisfactory harvesting efficiency makes them incompetent. The need for higher efficiency becomes crucial for lower power phones or when the application requires more than one sensor [7]. Thirdly, during the measurements, audio files were played on the local media player to output the required tone. Depending on the phone, it is also possible to use customized apps to generate higher voltage tones compared to playing a standard audio file.

TABLE II. SUMMARY OF POWER REQUIREMENTS OF VARIOUS MEDICAL DEVICES

\begin{tabular}{|l|l|c|}
\hline \multicolumn{1}{|c|}{ Instrumentation } & \multicolumn{1}{c|}{ Author (year) } & Power [mW] \\
\hline Pulse Oximetry & Tavakoli et al. (2010) [8] & 4.8 \\
\hline Bio-impedance & Lee et al. (2013) [9] & 7.5 \\
\hline Skin pH & Bandodkar et al. (2013) [10] & 5 \\
\hline Galvanic skin sensor & Fulford-Jones et al (2004) [11] & 2 \\
\hline Potentiostat & Sun et al. (2014) [12] & 6.9 \\
\hline ECG & Firth et al. (1995) [13] & 30 \\
\hline Immunoassay & Laksanasopin et al. (2015) [4] & 1.6 \\
\hline
\end{tabular}

\section{HARVESTING CIRCUIT}

The power harvesting circuit design discussed here is inspired by the topology from University of Michigan's "Hijack" project [3]. It uses a micro-transformer to step up a low voltage tone from the phone. The tone is rectified by a high efficiency rectifier [14] and passed through a Schottky diode to block reverse current. The diode can then be followed by a lowdropout regulator (LDO) to regulate the rectified signal to a desired value [15]. This is desirable when the rectified voltage is close to the final supply voltage $\left(V_{\mathrm{DD}}\right)$ of the external sensor circuit. In some cases where the rectified voltage is substantially higher than the final $V_{\mathrm{DD}}$, using an LDO alone dramatically reduces the overall efficiency of the power harvester and limits the achievable output power. This efficiency drop is due to the fact that the power loss of the LDO is proportional to the voltage difference between the input and output. Hence, when the rectified voltage is much larger than the desired supply voltage, due to a low supply voltage or a large ratio transformer, a DCDC converter is placed between the rectifier and the LDO [16]. These two cases will be both addressed in more detail in the next few sections.

However, even with a high efficiency rectifier and the addition of a DC-DC converter to reduce loss, this topology can still suffer from overall low efficiency due to the impedance mismatch between the harvester and a smartphone. While this basic design will work for a subset of mobile phones with powerful audio ports, it will not be able to guarantee enough power from all phones. Hence, in order to be able to interface a sensor with a much wider set of mobile phones, an impedance matching network (Fig. 3) that helps achieve efficient power

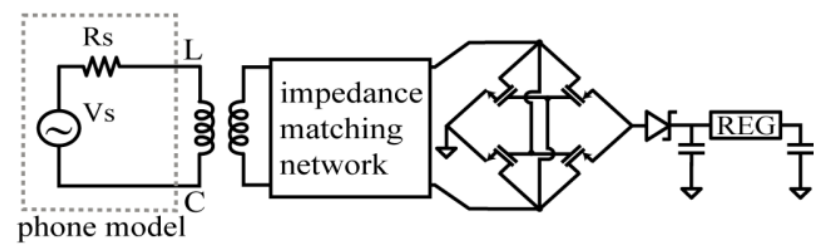

Fig. 3. Power harvesting circuit. 
transfer is added. To understand how this matching network works, the phone can be modelled as a voltage source $V_{\mathrm{S}}$ with a source impedance $R_{\mathrm{S}}$ using the measured results in Section II. In order to extract the most energy, $R_{\mathrm{S}}$ should match the input impedance of the power harvester. Due to the transformer, the input impedance of the power harvester is dependent on frequency. However, as confirmed by the measured data, this impedance only matches the phone's output impedance (2-4 $\Omega$ ) at frequencies that are much higher than $20 \mathrm{kHz}$, beyond the limit of most phones. The purpose of the added matching network is to shift the frequency range of optimal matching down below $20 \mathrm{kHz}$ to frequency values the phone can output. Once the impedance has moved to this approximate range, any mobile phone can then adjust its output frequency to find an optimal power transfer point. In order to properly design this matching network, both the source impedance of the phone and the input impedance of the harvester must be carefully explored. The first step in this process is to model the load and work backwards to the input side of the harvester.

\section{A. Modeling the Peripheral Device}

An accurate model of the load after the active rectifier is important in deriving the input impedance of the harvester. Without loss of generality, we model the sensor circuit as a resistance $R_{\text {EXT. }}$. As shown in Fig. 4, the load seen by the active rectifier is $R_{\text {rec}}$. The inclusion of the DC-DC converter changes this impedance, therefore the two situations are discussed separately.

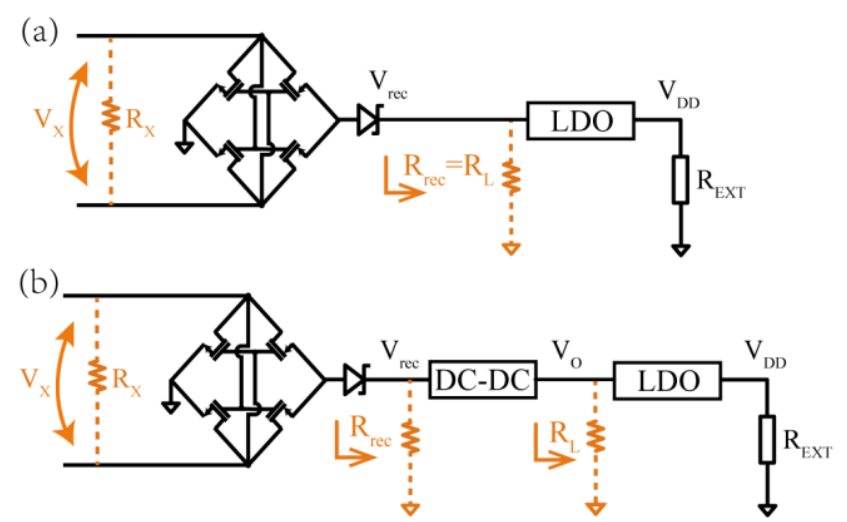

Fig. 4. Two cases in calculating the impedance seen by the rectifier, $R_{\text {rec }}$. In (a), only an LDO is used between the rectifier and the sensor. In (b), a DC-DC converter is added betwwen the rectifier and the LDO.

Case 1) When only an LDO is needed

Depending on the application, a designer may have the freedom to set $V_{\mathrm{DD}}$ close to $V_{\text {rec. If }} V_{\text {rec }}$ is just slightly above $V_{\mathrm{DD}}$, only an LDO is needed to ensure a stable and robust supply, as illustrated in Fig. 4 (a). The LDO and the rest of the circuitry can be modeled as a resistive load $R_{\mathrm{L}}$. In this case, $R_{\text {rec }}$ equals to $R_{\mathrm{L}}$.

\section{Case 2) Voltage Mismatch Considerations}

It is also possible that the rectified voltage is much higher than $V_{\mathrm{DD}}$, due to a high turn ratio transformer or a low $V_{\mathrm{DD}}$, in which case, a considerable portion of the power is lost through the regulation. In such cases, as Fig. 4 (b) shows, a DC-DC converter is added before the regulator to reduce the regulation loss. The DC-DC converter should have good efficiency within its nominal power range to improve upon the efficiency of the
LDO alone. The DC-DC converter alters $R_{\text {rec. }}$ In Fig. 4 (b), $V_{\text {rec }}$ is defined to be the voltage across the load $R_{\mathrm{rec}}$, and $V_{\mathrm{O}}$ is the voltage across $R_{\mathrm{L}}$. According to power conservation,

$$
\begin{gathered}
\frac{V_{r e c}^{2}}{R_{r e c}} \cdot \eta=\frac{V_{O}^{2}}{R_{L}}, \\
R_{r e c}=\eta \cdot R_{L} \cdot\left(\frac{V_{r e c}}{V_{O}}\right)^{2}=\frac{\eta R_{L}}{2 D^{2}}
\end{gathered}
$$

where $D$ is the voltage converting ratio, and $\eta$ denotes the power efficiency of the DC-DC converter (often > 80\%).

\section{B. Designing the Matching Network}

With the load properly modelled, the impedance of the harvester, which consist of the rectifier and the transformer, must now be considered in designing the matching network (Fig. 5). Unfortunately, it is not possible to calculate the exact input impedance of the active rectifier by hand due to the high nonlinearity of the transistors. Instead, conservation of power can be used to determine an average estimate of the impedance. Firstly, considering that this active rectifier itself has been shown to be very efficient [5], the rectification loss is assumed to be negligible. This approximation has been confirmed with lab measurements by observing the voltage drop over the transistors and Schottky diode $(\sim 200 \mathrm{mV})$ to be much smaller than the rectified voltage $(20-30 \mathrm{~V})$. By assuming no loss in the rectification, the following power equation can be used to determine the impedance of the rectifier:

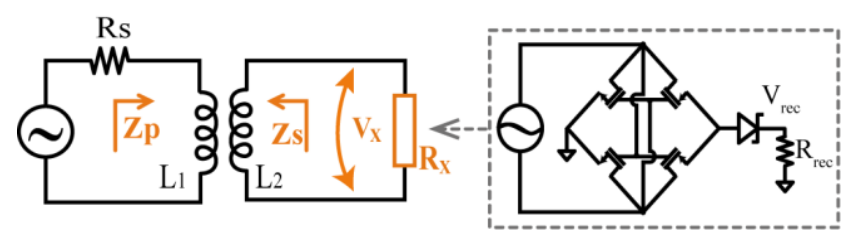

Fig. 5. Equivalent circuit model of the active rectifier

$$
P_{I N}=\frac{V_{X, R M S}^{2}}{R_{X}}=P_{O U T}=\frac{V_{r e c}^{2}}{R_{r e c}}
$$

where $P_{\mathrm{OUT}}$ is the power delivered to $R_{\mathrm{rec}}$ and $P_{\mathrm{IN}}$ is the power entering the rectifier, $V_{\mathrm{X}}$ is the AC voltage between the two terminals of the rectifier, and $R_{\mathrm{X}}$ is the equivalent impedance of the rectifier seen by the transformer. Hence, neglecting the voltage drop over the rectification, the input impedance of the active rectifier is expressed as:

$$
R_{X}=\frac{1}{2} R_{\text {rec }}
$$

There is also a small $(\sim \mathrm{fF})$ parallel capacitance, however it is insignificant in this frequency range. The impedance seen looking into the micro-transfer includes the inductance $L_{1}$ (or $L_{2}$ ) as well as the impedance reflected from the other side, depending on which direction you look. The impedances seen from the primary side, $Z_{\mathrm{P}}$, and the secondary side, $Z_{\mathrm{S}}$, are:

$$
\begin{gathered}
Z_{p}=j \omega L_{1}+\frac{\omega^{2} M^{2}}{R_{X}+j \omega L_{2}} \\
Z_{s}=\frac{\omega^{2} M^{2}}{R s+j \omega L_{1}}+j \omega L_{2} \\
M=k \sqrt{L_{1} L_{2}}
\end{gathered}
$$


where $M$ is the mutual inductance, and $k$ is the coupling coefficient.

There are several possible topologies that can be used. Two different configurations of the network are compared in Fig. 6 with the network placed on either side of the transformer. In both configurations, $R_{1}$ and $R_{2}$ are the DC resistance of the transformer, which, with such small source impedances, are not negligible. $Z_{1}$ and $Z_{2}$ are the impedances seen from the points drawn. $X_{\mathrm{S}}$ and $X_{\mathrm{P}}$ represent the reactance added to the network.

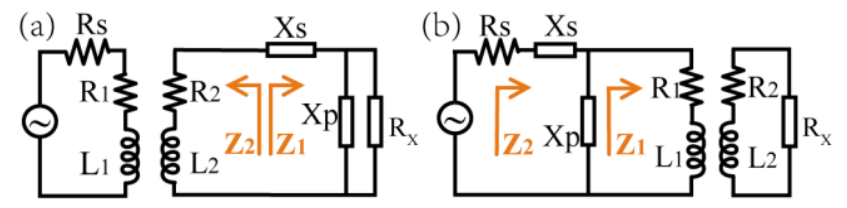

Fig. 6. Impedance matching configurations. In (a), the impedance matching network coms before the transformer and in (b) it comes after the transformer.

The goal is to match the load with the source impedance. Hence, $Z_{1}$ must be matched with $Z_{2}$ in Fig. 6 (a) and $Z_{2}$ with $R_{\mathrm{S}}$ in Fig. 6 (b). Configuration (a) is presented in this work. This is because in (b), even though $R_{\mathrm{S}}$ and $Z_{2}$ are matched, there is loss through $R_{1}$ and $R_{2}$, because $R_{\mathrm{X}}$ may still be unmatched with the impedance looking into the transformer. Whereas in (a), $R_{1}$ and $R_{2}$ are lumped into $R_{\mathrm{S}}$ in matching, and $R_{\mathrm{X}}$ is matched with the impedance looking into the transformer, ensuring maximum power is delivered to $R_{\mathrm{X}}$.

In Fig. 6 (a),

$$
\begin{gathered}
Z_{1}=j X s+R_{X} \| j X_{P}, \\
\text { and } \quad Z_{2}=\frac{\omega^{2} M^{2}}{R s+R_{1}+j \omega L_{1}}+j \omega L_{2}+R_{2} .
\end{gathered}
$$

After expanding, and rearranging terms,

$$
Z_{1}=\frac{R_{X} X_{P}^{2}}{X_{P}^{2}+R_{X}^{2}}+j\left(\frac{R_{X}^{2} X_{P}}{X_{P}^{2}+R_{X}^{2}}+X_{S}\right) .
$$

Finally, by setting $Z_{1}=Z_{2}{ }^{*}$, the equation yields solutions for $X_{\mathrm{P}}$ and $X_{\mathrm{S}}$, giving us a set of solutions to implement this network. Next, the many combinations of inductors and capacitors that satisfy these conditions will be discussed.

\section{Topology compraison}

Regardless of the topologies discussed below, the matching network is designed around an $R_{\mathrm{S}}$ of $3 \Omega$, the average source resistance of most phones. The natural resonance frequency is set to be $15 \mathrm{kHz}$, because this point strikes a good balance between the transformer performance and $R_{\mathrm{S}}$ tolerance. At this frequency, the transformer has low loss through the DC resistance, and there is still enough frequency room to adjust for different $R_{\mathrm{S}}$ values.

To elucidate the concept of the matching network assume, $R_{\mathrm{L}}=1.2 \mathrm{k} \Omega$. For case 1 ), where only an LDO is used, by following the calculations, two impedance matching topologies are explored. In Fig. 7, both designs use a 1:20 turn ratio microtransformer. Topology (a) uses a standard LC network, but requires a $16 \mathrm{mH}$ inductor. This is not desirable because such an inductor is quite large. Alternatively, the impedance matching network can also be implemented using two capacitors, as
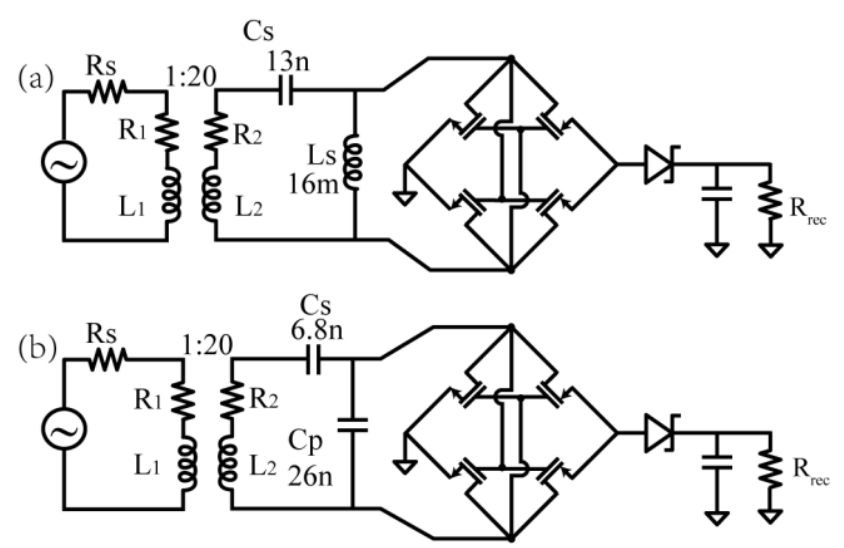

Fig. 7. Impedance matching topologies for case 1.

topology (b) shows. This design leverages the secondary inductance to match with the source impedance eliminating the need for a discrete inductor. Both Fig. 7 (a) and Fig. 7 (b) have been simulated to have efficiencies of $87.1 \%$ and $87.93 \%$ respectively. Since topology (b) has a slightly better efficiency and, more importantly, obviates the need of a large inductor, it is chosen to be the topology used for case 1).

For case 2), it can be derived from equation (2) and (4) that

$$
R_{X}=\frac{\eta R_{L}}{4 D^{2}}
$$

Applying equation (10) with this new load generates a different set of capacitor values for topology (b) as shown in Fig. 8. In order to keep both $X_{\mathrm{S}}$ and $X_{\mathrm{P}}$ capacitive, a 1:50 turn ratio transformer is used because this new transformer has a higher inductance on the secondary side. Simulations show that this topology with the added DC-DC converter has an efficiency of $81.88 \%$. Compared to the efficiency result in case 1), although the efficiency is a little lower, the designer has more freedom in choosing $V_{\mathrm{DD}}$.

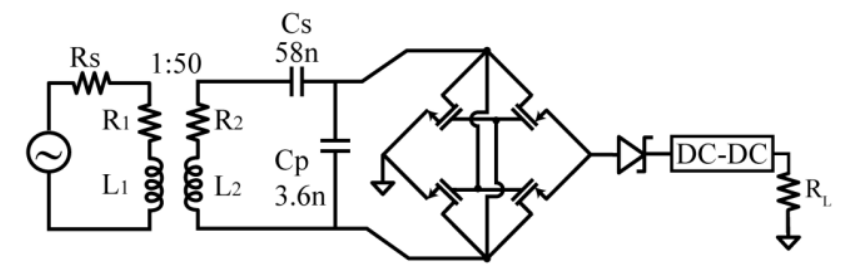

Fig. 8. Impedance matching topology for case 2.

\section{Maximum Power Point Tracking}

Because of the differences in phone output impedance, each phone model has a unique audio output frequency at which the system has its optimal efficiency. It is crucial to find the optimal frequency for each phone in order to ensure that the device is compatible with any mobile phone. A Maximum Power Point 


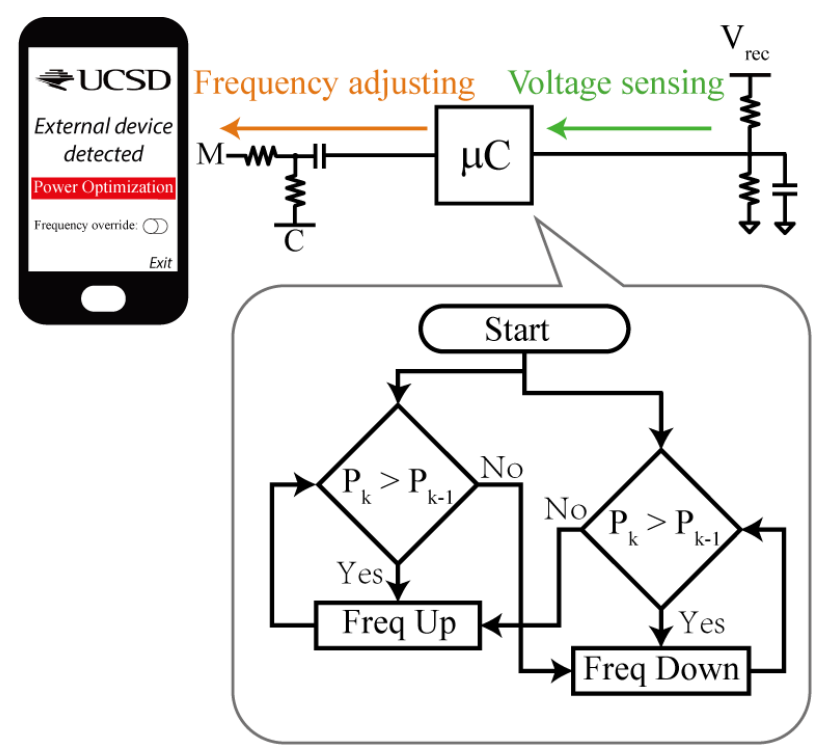

Fig. 9. MPPT machenism is added to find the optimal frequency for each phone.

Tracking algorithm [17], [18] is designed to keep track of the optimal power point and dynamically adjust the frequency. Fig. 9 illustrates a MPPT circuit schematic and a software algorithm flowchart. Since the load after the rectifier is resistive, $V_{\text {rec }}$ is proportional to the output power. Therefore, this voltage is sensed and fed back into the mobile phone. A microprocessor samples $V_{\text {rec}}$, and also computes how frequency should be adjusted. This command is transmitted over the microphone channel into the mobile phone. The left branch of the flowchart increases the frequency of the tone to reach the maximum power point, while the right branch prevents the frequency to go beyond this point. When the maximum power point is reached, it will oscillate around the optimal point. If the step size $k$ is chosen to be small enough, this state can be seen as the optimal point. This MPPT algorithm is run before the actual test when the device first connects to a phone. It ensures that the device has optimal power output regardless of the phone model.

\section{SIMULATIONS AND MEASUREMENTS}

So far, two topologies (Fig. 7b, and Fig. 8) have been presented for two different scenarios. Considering that case 2) is suitable for more applications, tests in this section are based on

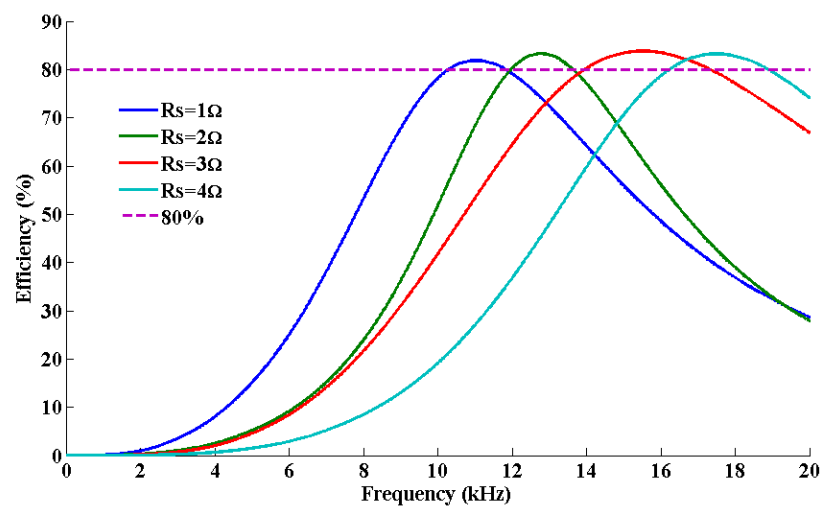

Fig. 10. Simulated efficiency for different phones.

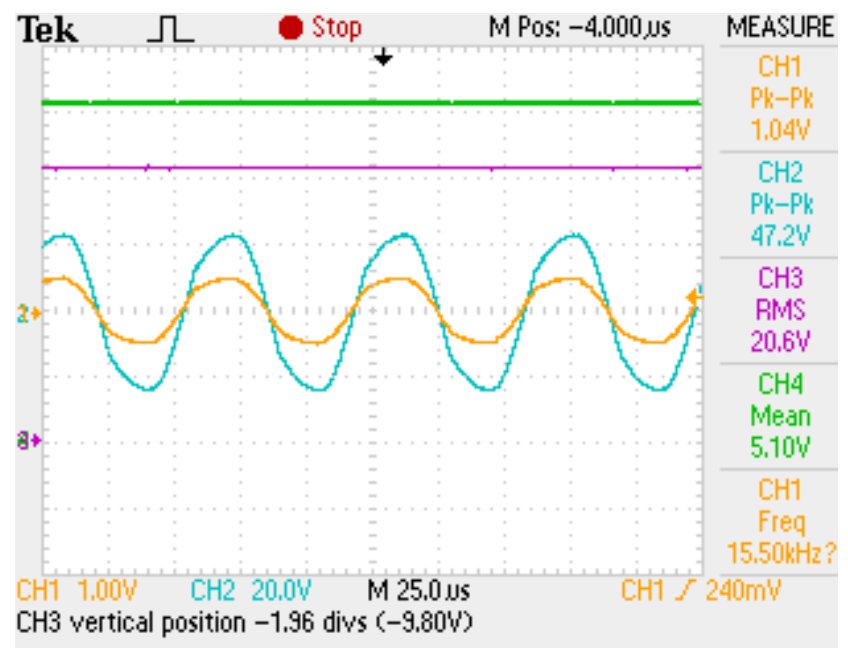

Fig. 11. Oscilloscope screenshot of the presented circuit. $\mathrm{CH} 1$ shows the audio signal from the phone. $\mathrm{CH} 2$ shows the output of the transormer. $\mathrm{CH} 3$ shows the rectified voltage $\left(\mathrm{V}_{\text {rec }}\right)$, and $\mathrm{CH} 4$ shows the uregulated $\mathrm{V}_{\mathrm{DD}}$.

this design. For most popular smartphones, $R_{\mathrm{S}}$ varies from $1 \Omega$ to $4 \Omega$. Simulations (Fig. 10) show that with the DC-DC converter, the presented circuit has a good tolerance of varying $R_{\mathrm{S}}$ values. With different peak frequencies, phones are able to output higher than $80 \%$ of the available power within the frequency range.

To further evaluate the presented design, the topology in Fig. 8 was implemented on a PCB with $V_{\mathrm{DD}}$ set to be $5 \mathrm{~V}$. A tone is generated by an iPhone $4 \mathrm{~S}$ and the frequency was swept to determine the optimal efficiency point. Fig. 11 shows a screenshot of the oscilloscope probing the circuit in operation. The rectified voltage $(\mathrm{CH} 3)$ of $20 \mathrm{~V}$ is converted to $5 \mathrm{~V}(\mathrm{CH} 4)$ via the DC-DC. The maximum output power was measured to be $18.7 \mathrm{~mW}$ (77.9\% efficiency).

To compare the presented circuits with previous designs, we have measured maximum output power and efficiency across the signal frequency for each (Fig. 12 and Table III). With the impedance matching network, the topology for case 1 achieves $85.3 \%$ of the available power at $15.5 \mathrm{kHz}$; with the addition DCDC converter, the maximum efficiency is $79 \%$ at $15 \mathrm{kHz}$. Without dedicated impedance matching, the highest efficiency of the Hijack circuit is $47 \%$ at $20 \mathrm{kHz}$. The NXP Quick Jack uses

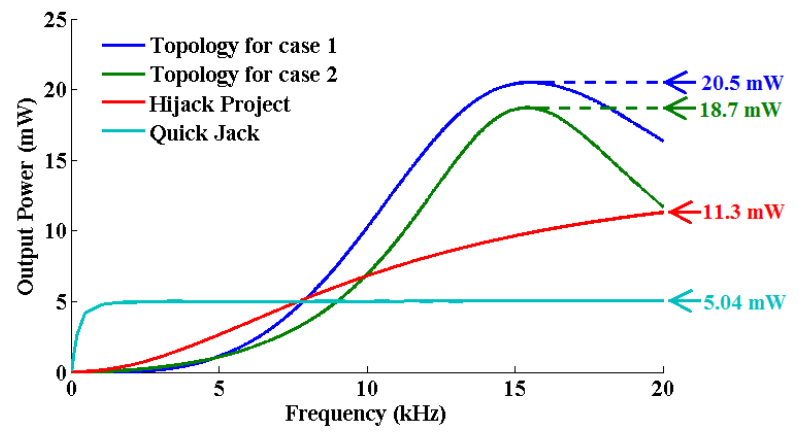

Fig. 12. Comparison of the output curves measured from the presented circuit (blue), and prior art. 
a diode based voltage multiplier technique to boost and rectify the tone. The efficiency is less dependent on the signal frequency, however it suffers from a severe loss over the Schottky diodes and can only achieve $21 \%$. The design proposed in [6] is an integrated solution to rectify and regulate the voltage. However, without more detailed information on this circuit, it is impossible to obtain comprehensive efficiency data on this design. From the description, a 1:2 transformer is needed to boost the audio signal before rectifying by the proposed circuit. Due to the low quality factor associated with such low turn ratio transformer and the impedance mismatch between the phone and

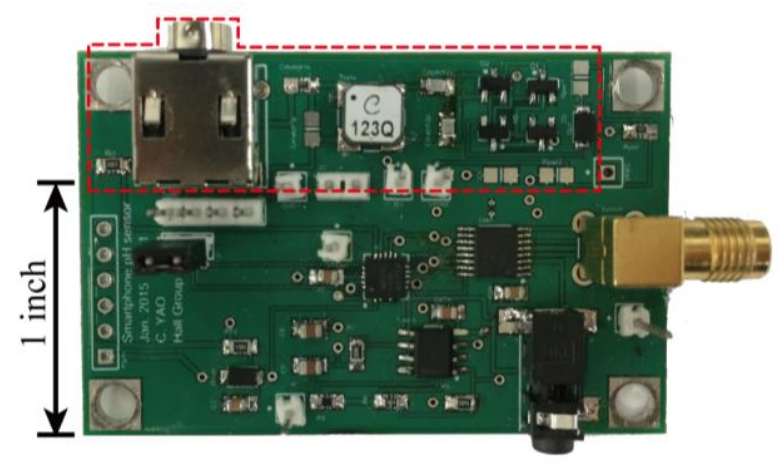

Fig. 13. A mobile phone-based $\mathrm{pH}$ sensor prototype designed based on the presented topology. Area in red dash box is the power harvesting portion.

the transformer, the loss during this process is significant. As a comparison, our circuit addresses this issue by using a high turn ratio transformer and an impedance matching network, resulting in a superior overall efficiency. A mobile phone-based $\mathrm{pH}$ sensor is designed and implemented based on this schematic (Fig. 13). With right and mic channels left for communication, it can be both powered and controlled easily by the mobile phone.

TABLE III. TOPOLOGY COMPARISON

\begin{tabular}{|l|l|l|l|}
\hline \multicolumn{1}{|c|}{ Topology } & $\begin{array}{c}\text { Simulation } \\
\text { Efficiency }\end{array}$ & $\begin{array}{c}\text { Measured } \\
\text { Efficiency }\end{array}$ & \multicolumn{1}{|c|}{ Sensitivity } \\
\hline Case 1 & $87.93 \%$ & $85.4 \%$ & $\begin{array}{l}\text { Sensitive to frequency } \\
\text { and source impedance }\end{array}$ \\
\hline Case 2 & $81.88 \%$ & $77.9 \%$ & $\begin{array}{l}\text { Less sensitive to } \\
\text { frequency, not sensitive } \\
\text { to source impedence }\end{array}$ \\
\hline $\begin{array}{l}\text { Hijack } \\
\text { project }\end{array}$ & $52 \%$ & $47 \%$ & $\begin{array}{l}\text { Insensitive to both } \\
\text { frequency and source } \\
\text { impedance }\end{array}$ \\
\hline $\begin{array}{l}\text { NXP Quick } \\
\text { Jack }\end{array}$ & $21 \%$ & N/A & \\
\hline
\end{tabular}

\section{CONCLUSION AND FUTURE WORK}

We have implemented a power harvesting circuit that can be used to efficiently harvest power from the smartphone's audio port for external mHealth oriented devices. The use of the standard audio port makes this design compatible across different models and makes of phones. Mobile computing is a rapidly developing technology and this power harvesting circuit can enable portable medical applications. Within foreseeable future, we hope to be able to transform every mobile device into a powerful all-in-one diagnostic and health tool.

\section{REFERENCES}

[1] "2 Billion Consumers Worldwide to Get Smart(phones) by 2016 eMarketer." [Online]. Available: http://www.emarketer.com/Article/2Billion-Consumers-Worldwide-Smartphones-by-2016/1011694. [Accessed: 30-May-2015].

[2] A. Giridharadas, "Where a Cellphone Is Still Cutting Edge," The New York Times, 10-Apr-2010.

[3] Y.-S. Kuo, T. Schmid, and P. Dutta, "Hijacking Power and Bandwidth from the Mobile Phone's Audio Interface," presented at the First Annual Symposium on Computing for Development (DEV'10), 2010.

[4] T. Laksanasopin, T. W. Guo, S. Nayak, A. A. Sridhara, S. Xie, O. O. Olowookere, P. Cadinu, F. Meng, N. H. Chee, J. Kim, C. D. Chin, E. Munyazesa, P. Mugwaneza, A. J. Rai, V. Mugisha, A. R. Castro, D. Steinmiller, V. Linder, J. E. Justman, S. Nsanzimana, and S. K. Sia, “A smartphone dongle for diagnosis of infectious diseases at the point of care," Sci. Transl. Med., vol. 7, no. 273, pp. 273re1-273re1, Feb. 2015.

[5] "Quick-Jack - the multi-purpose, self-powered data port - NXP Semiconductors." [Online]. Available: http://www.nxp.com/campaigns/lpc800-go/quick-jack.

[6] S. Verma, A. Robinson, and P. Dutta, "AudioDAQ: Turning the Mobile Phone's Ubiquitous Headset Port into a Universal Data Acquisition Interface," in Proceedings of the 10th ACM Conference on Embedded Network Sensor Systems, New York, NY, USA, 2012, pp. 197-210.

[7] A. Nemiroski, D. C. Christodouleas, J. W. Hennek, A. A. Kumar, E. J. Maxwell, M. T. Fernández-Abedul, and G. M. Whitesides, "Universal mobile electrochemical detector designed for use in resource-limited applications," Proc. Natl. Acad. Sci., vol. 111, no. 33, pp. 11984 11989, Aug. 2014.

[8] M. Tavakoli, L. Turicchia, and R. Sarpeshkar, “An Ultra-Low-Power Pulse Oximeter Implemented With an Energy-Efficient Transimpedance Amplifier," IEEE Trans. Biomed. Circuits Syst., vol. 4, no. 1, pp. 27-38, Feb. 2010.

[9] S. Lee, S. Polito, C. Agell, S. Mitra, R. F. Yazicioglu, J. Riistama, J. Habetha, and J. Penders, "A Low-power and Compact-sized Wearable Bio-impedance Monitor with Wireless Connectivity," J. Phys. Conf. Ser., vol. 434, no. 1, p. 012013, Apr. 2013.

[10] A. J. Bandodkar, V. W. S. Hung, W. Jia, G. Valdés-Ramírez, J. R. Windmiller, A. G. Martinez, J. Ramírez, G. Chan, K. Kerman, and J. Wang, "Tattoo-based potentiometric ion-selective sensors for epidermal pH monitoring," The Analyst, vol. 138, no. 1, pp. 123-128, Jan. 2013.

[11] T. R. F. Fulford-Jones, G.-Y. Wei, and M. Welsh, "A Portable, LowPower, Wireless Two-Lead EKG System," Proc. 26th Annu. Iternational Conf. IEEE EMBS, no. September 1-5, 2004.

[12] A. Sun, T. Wambach, A. G. Venkatesh, and D. A. Hall, "Live demonstration: A low-cost smartphone-based electrochemical biosensor for point-of-care diagnostics," in 2014 IEEE Biomedical Circuits and Systems Conference (BioCAS), 2014, pp. 190-190.

[13] J. Firth and P. Errico, "Low-Power, Low-Voltage IC choices for ECG System Requirements," Analog Dialogue, no. 29-3.

[14] K. Kotani, A. Sasaki, and T. Ito, "High-Efficiency Differential-Drive CMOS Rectifier for UHF RFIDs," IEEE J. Solid-State Circuits, vol. 44, no. 11, pp. 3011-3018, Nov. 2009.

[15] C.-H. Hsieh, C.-Y. Du, and S.-Y. Lee, "Power management with energy harvesting from a headphone jack," in 2014 IEEE International Symposium on Circuits and Systems (ISCAS), 2014, pp. 1989-1992.

[16] S.-E. Adami, N. Degrenne, C. Vollaire, B. Allard, and F. Costa, "Ultralow power, low voltage, autonomous resonant DC-DC converter for low power applications," in 2013 Fourth International Conference on Power Engineering, Energy and Electrical Drives (POWERENG), 2013, pp. 1222-1228.

[17] I. Lee, S. Bang, D. Yoon, M. Choi, S. Jeong, D. Sylvester, and D. Blaauw, "A ripple voltage sensing MPPT circuit for ultra-low power microsystems," presented at the VLSI Circuits (VLSIC), 2013 Symposium on, 2013, pp. C228-C229.

[18] K. K. Win, S. Dasgupta, and S. K. Panda, "An optimized MPPT circuit for thermoelectric energy harvester for low power applications," in 2011 IEEE 8th International Conference on Power Electronics and ECCE Asia (ICPE ECCE), 2011, pp. 1579-1584. 